Tohoku J. Exp. Med., 1995, 175, 219-223

\title{
A Simple Maneuver in Follow-Up Digital Subtraction Angiography for Multiple Coronary-Aorta Bypass Grafts
}

\author{
Hideaki Mitsui, Masayuki Zuguchi*, Shoki Takahashi, \\ Izuru Yoshida $\dagger$, Shin Maruoka, Yoshihisa Shimanuki, \\ Akiniro Satoh and Kiyohiko Sakamoto \\ Department of Radiology, Tohoku University School of \\ Medicine, Sendai 980-7\%, *Department of Radiology, \\ Tohoku Medical Junior College, Sendai 980-77, and \\ $\dagger$ Department of Cardiovascular Surgery, Tohoku University \\ School of Medicine, Sendai 980-77
}

Mitsui, H., Zuguchi, M., Takahashi, S., Yoshida, I., Maruoka, S., Shimanuki, Y., Satoh, A. and Sakamoto, K. A Simple Maneuver in Follow-Up Digital Subtraction Angiography for Multiple Coronary-Aorta Bypass Grafts. Tohoku J. Exp. Med., 1995, 175 (4), 219-223 — A simple maneuver in intraarterial digital subtraction angiography (DSA) is proposed to improve the images of left internal thoracic artery bypass grafts (LITAGs). A contrast flush in the left subclavian artery, using a catheter that had been percutaneously introduced from the left brachial artery, was carried out in 14 patients with multiple coronary-aorta bypass grafts (CABGs) after aortic DSA. The side-hole portion of the catheter tip was positioned in the proximal left subclavian artery as the coiled portion remained in the aortic arch. Nonionic contrast medium $(350 \mathrm{mg} / \mathrm{ml}$ of iodine) was injected with an automatic injector in a volume of 6-8 $\mathrm{ml}$ and at a flow rate of 3$4 \mathrm{ml} / \mathrm{sec}$. Left subclavian arterial flush DSA delineated the LITAGs excellently in 11 , well in 3 , and fairly or poorly in none of the 14 cases. There were no complications in any of these cases. A supplemental contrast flush of the left subclavian artery with a pigtail catheter following aortic DSA improves the diagnosis of multiple $\mathrm{CABG}$ patency.—- coronary aorta bypass grafts (CABGs); intra-arterial digital subtraction angiography (IA-DSA)

After coronary-aorta bypass graft (CABG) surgery, intraarterial digital subtraction angiography (DSA) through a transbrachial approach has been used for the evaluation of graft patency (Martin and Charles 1986; Richard and Donald 1990; Terada et al. 1990; Mancini 1991). Although aortic DSA has delineated saphenous vein grafts (SVGs) well, it does not clearly define left internal thoracic artery bypass grafts (LITAGs), which have been commonly used in current CABG

Received June 22, 1994; revision accepted for publication January 31, 1995.

Address for reprints: Hideaki Mitsui, M.D., Division of Radiology, Takeda General

Hospital, 3-11 Yamaga-machi, Aizu-Wakamatsu, Fukushima 965, Japan. 
surgery. To improve the image of LITAGs, we added a process of flushing the left subclavian arteries with contrast medium. The diagnostic advantage of a subclavian arterial flush DSA is reported below.

\section{Materials and Methods}

DSA by contrast flush in the left subclavian artery was performed in 14 patients with multiple CABGs, using a 4F-pigtail catheter percutaneously introduced from the left brachial artery. The patients' ages ranged from 45 to 77 years (mean, 61). Following aortic DSA to obtain SVG imaging, the catheter was subsequently pulled back to a spot where the side-hole part of the catheter was positioned in a proximal portion of the left subclavian artery when the coiled catheter tip remained in the aortic arch (Fig. 1). A non-ionic contrast medium of Iodine (350 mg/ml of iodine: Optiray 350, Mallinckrodt, St. Louis, MO, USA) was injected with an automatic injector in a volume of 6-8 $\mathrm{ml}$ and at a flow rate of 3$4 \mathrm{ml} / \mathrm{sec}$. DSA was performed using a Digitron (Siemens, Erlangen, Germany) with an 11-inch image intensifier with a $512 \times 512$ matrix. Three experienced

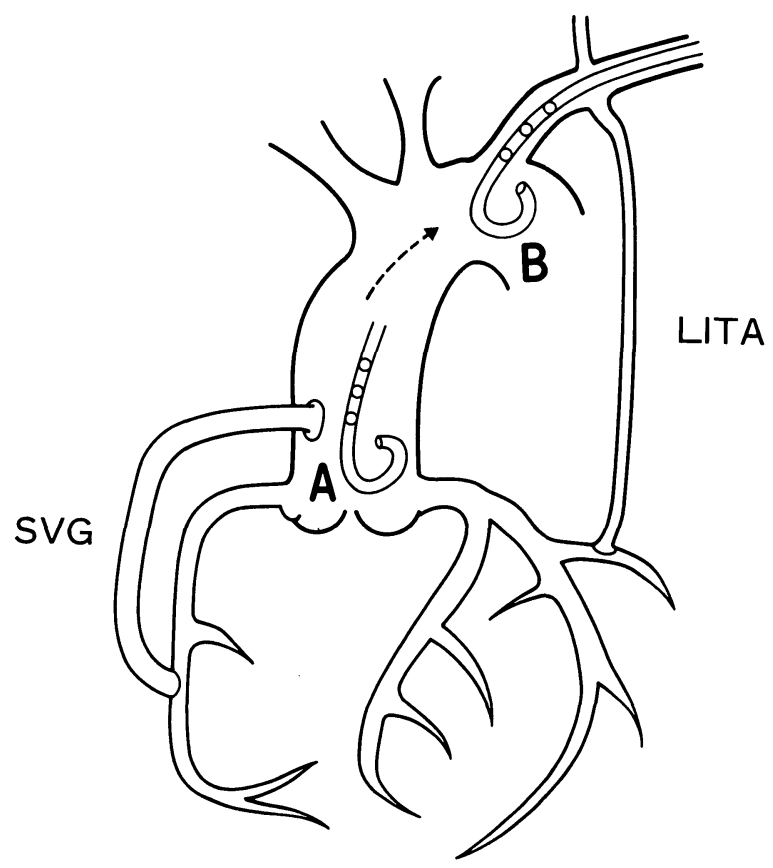

Fig. 1. Diagram illustrating catheter manipulation.

A. Position of the pigtail catheter for aortic DSA to obtain SVG imaging.

B. Position of the pigtail catheter for LITAG imaging. The catheter is pulled back to a position, by which the side-hole portion of the catheter is positioned in the proximal left subclavian artery as the coiled catheter tip remaining in the aortic arch. SVG, saphenous vein bypass graft; LITA, left internal thoracic artery graft. 
radiologists subjectively graded the image quality of the left internal thoracic artery grafts (LITAGs) according to four categories (excellent: good delineation of the LITAGs and the anastomotic portion; good: good delineation of the LITAGs with poor depiction of the anastomotic portion; fair: fair delineation of the LITAGs without depiction of the anastomosed coronary artery; and poor: no delineation of the LITAGs).

\section{Results}

Left subclavian arterial flush DSA at a low dose and a low flow rate contrast injection favorably depicted LITAGs when compared with aortic DSA (Table 1). Excellent delineation of the LITAGs was achieved in 11, good in 3, and fair or poor in none of the 14 cases that we examined. We could evaluate not only the patency but also intraluminal changes of the grafts and anastomosed coronary arteries by this method. The LITAGs were patent without stenosis in 8, patent but with a narrowed segment in 5, and occluded in one (Fig. 2). No complications occurred in any case.

\section{Discussion}

Either CT, intravenous DSA, or aortic DSA has been generally undertaken to
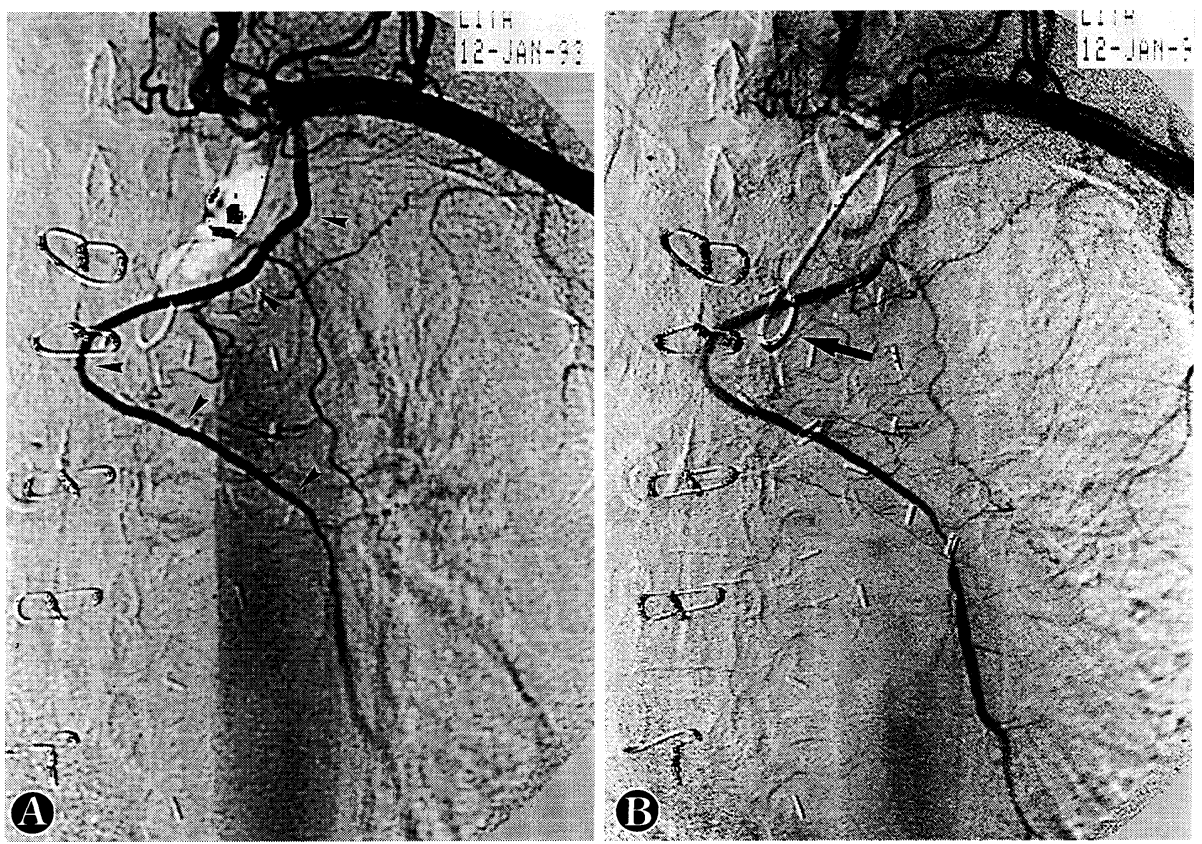

Fig. 2. Left subclavian flush DSA for LITAG imaging in a 53-year-old male.

A. Early phase image; B. Later phase image.

The LITAG is clearly delineated (arrowheads).

Note that coiled portion of the catheter tip remained in the aorta (arrow). 
TABLE 1. Results of DSA for multiple CABGs in 14 cases

\begin{tabular}{cccccl}
\hline & \multicolumn{5}{c}{ Image quality of the LITAG } \\
\hline $\begin{array}{c}\text { Case } \\
\text { No. }\end{array}$ & Sex & $\begin{array}{c}\text { Age } \\
\text { (years) }\end{array}$ & $\begin{array}{c}\text { Aortic } \\
\text { flush }\end{array}$ & $\begin{array}{c}\text { Subclavian } \\
\text { flush }\end{array}$ & \multicolumn{1}{c}{ Findings of LITAG } \\
\hline 1 & M & 52 & Poor & Excellent & Stenosis at the anastomosis \\
2 & F & 62 & Fair & Excellent & Severe stenosis at the anastomosis \\
3 & F & 45 & Poor & Good & Severe stenosis at the anastomosis \\
4 & M & 53 & Poor & Excellent & No stenosis \\
5 & F & 75 & Fair & Excellent & $25 \%$ stenosis at the distal LITA \\
6 & M & 60 & Fair & Excellent & No stenosis \\
7 & M & 66 & Fair & Excellent & No stenosis \\
8 & F & 53 & Fair & Excellent & No stenosis \\
9 & M & 67 & Poor & Excellent & No stenosis \\
10 & F & 70 & Fair & Good & Suspicious of occlusion at the anastomosis \\
11 & M & 60 & (-) & Excellent & No stenosis \\
12 & F & 77 & Good & Excellent & No stenosis \\
13 & F & 64 & Poor & Good & Stenosis at the anastomosis \\
14 & M & 51 & Fair & Excellent & No stenosis \\
\hline
\end{tabular}

CABGs, coronary-aorta bypass grafts ; LITAG, left internal thoracic artery bypass graft; (-), aortography unavailable.

follow-up CABG patency (Martin and Charles 1986; Richard and Donald 1990; Terada et al. 1990; Mancini 1991). Since the advent of LITAGs, however, those modalities have been found to be less than satisfactory for delineating grafts. The SVG anastomosed to the ascending aorta is well opacified by aortic DSA, while the patency of LITAGs may not be evaluated accurately because a sufficient concentration of the contrast medium may not be achieved in the LITA. In this situation, only intraarterial DSA has a potential to get good opacification of LITAGs by a semi-selective technique (Table 1).

We therefore modified the conventional method in the following manner: by introducing a pigtail catheter from the left brachial artery, we performed aortic DSA and semi-selective DSA of LITAGs without a catheter change. This simple maneuver has not been reported in the literature.

Slow injection efficiently delivers a contrast medium through the side holes of a catheter that has been placed in the proximal subclavian artery with minimum delivery of the contrast medium through the end hole. The subclavian artery and the LITAG would thereby be sufficiently opacified. With this technique, catheter exchange is obviated. Furthermore, slow injection of the contrast medium presumably augments the safety of our method. However, the coiled part of the catheter tip should be carefully positioned in the aortic arch to avoid subintimal injection or cerebrovascular complications due to intimal scratching by the catheter tip (Richard and Donald 1990). 
In conclusion, a combination of aortic DSA and left subclavian flush DSA, using a thin pigtail catheter, is useful for follow-up of multiple CABG patency, including LITAGs. The left brachial approach and positioning of the catheter are essential for this study. The methodology is simple, safe, and cost-and time-effective.

\section{References}

1) Mancini, G.B.J. (1991) Applications of digital angiography to coronary circulation. In: Cardiac Imaging, edited by LM. Meluin, W.B. Saunders Company, Philadelphia, pp. 324-325.

2) Martin, J.L. \& Charles, B.H. (1986) Cardiovascular imaging: CT, MRI, DSA. In: Diagnostic Radiology, vol. 1, edited by G.G. Ronald \& J.A. David, Churchill Livingstone, New York, pp. 450-451.

3) Richard, E.K. \& Donald, S.B. (1990) Internal mammary angiography: A review of technical issues and newer methods. Cathet. Cardiovasc. Diagn., 20, 10-16.

4) Terada, Y., Suma, H., Saito, T., Fukuda, S., Takayama, T., Wanibuchi, Y. \& Furuta, S. (1990) Long-term results of coronary artery bypass grafting: Comparison of angiographic evaluation of internal thoracic artery and saphenous vein grafts. Kyobu-Geka, 43, 873-877. (in Japanese) 